\title{
VCOT Resident Publication Award
}

As the year came to a close, we had the difficult, but enjoyable, task of selecting the VCOT Resident Publication Award for 2017. This award, sponsored by Schattauer Publishers and VCOT, is given in recognition of the valuable contribution that our residents make to research in our field. To be eligible for the award, the senior author must have been enrolled in an accredited residency program at the time of submission of the paper to the journal. Only papers that were published under the classification of Original Research or Clinical Communication were considered for the award.

There were 10 eligible resident papers published in VCOT in 2017. Two came from the UK, one from Europe, one from Australia and six from the USA. They covered a diverse array of topics - small plates for radius fractures, radiographic scoring validation, implant mechanics, OA management, biomarkers of OA, vertebral pin placement, and TPLO outcomes. The papers were judged by a selection panel of four members of the Board of Reviewers of VCOT and the Deputy Editor. This was a very difficult task because the quality of all the papers was very high and each one received supportive comments from the judges.

It is a pleasure to announce that the winner for 2017 is Dr. Alen Lai from North Shore Veterinary Specialists in Sydney, Australia, for the paper entitled "Biomechanical comparison of pin and nitinol bone staple fixation to pin and tension band wire fixation for the stabilization of canine olecranon osteotomies" and co-authored with Chris Christou, Craig Bailey, Chris J. Tan, John Culvenor, Tian Wang, and William R. Walsh (1). Dr. Lai and colleagues showed that a nitinol staple was stiffer and stronger when compared to a standard tension band wire for olecranon osteotomy. The study was well designed and executed and the discussion addressed the findings and the limitations in a accurate and concise manner.

On behalf of VCOT and Schattauer Publishing who have very generously provided the award (€500 and a 1-year subscription to VCOT), we would like to offer our warm congratulations to Dr. Lai. We also want to say a sincere thank you to the judges who contributed to this important process. While it was a difficult task, it was also a great pleasure to see the amazing contributions that our young surgeons are making to the science of orthopaedics.

1. Lai A, Christou C, Bailey C, Tan CJ, Culvenor J, Wang T, Walsh WR. Biomechanical comparison of pin and nitinol bone staple fixation to pin and tension band wire fixation for the stabilization of canine olecranon osteotomies. VCOT 2017, 30 (4):324-330.

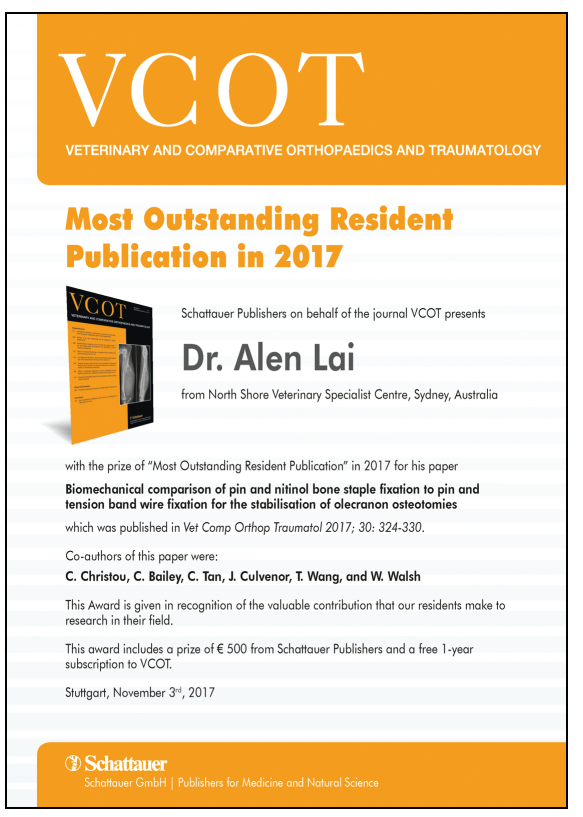

